\title{
Förster resonance energy transfer between quantum dots and dye immobilized in biopolymer particles
}

\author{
M.A. Gerasimova*, N.V. Slyusarenko, E.A. Slyusareva \\ Siberian Federal University, 79 Svobodny Prospect, 660041 Krasnoyarsk, Russia
}

Corresponding author: marina_2506@mail.ru

\begin{abstract}
We used high adsorption properties of the cationic biopolymer chitosan to synthesize colloidal polymer particles (average size about $0.3 \mu \mathrm{m}$ ) with immobilized CdTe quantum dots (QDs) and organic dye (erythrosin B). A high local concentration of fluorophores bound to the particles (about $10^{-3} \mathrm{M}$ ), as well as a wide overlap of their optical spectra result in an efficient (up to 80\%) Förster resonance energy transfer (FRET) from QDs ensembles to dye molecules. The FRET was registered by both steady-state (quenching of the donor and enhancement of the acceptor fluorescence) and time-resolved methods (decreasing of donor lifetime). The dependence of the transfer efficiency on acceptor concentration was analyzed within the scope of the Förster theory extended for the case of multiple energy transfer configuration. The average distances between the donor and acceptor as well as local concentration of fluorophores within particles were determined. It was demonstrated that the synthesized particles can be used as FRET-based sensitive probes for inter-fluorophore distance calculation within the range of $4 \div 9 \mathrm{~nm}$.
\end{abstract}

\section{INTRODUCTION}

Förster Resonance Energy Transfer (FRET) occurs between two molecular fluorophores - initially photoexcited donor (D) and acceptor (A) - without any intermediate emission of photons [1]. Two types of fluorophores, namely organic dyes and fluorescent proteins, were commonly used in the majority of the FRET-attributed studies. Several important conditions including an appropriate spectral overlap, alignment of two dipoles, sufficient quantum yield and lifetime of the donor, as well as the distance between the fluorophores $(r)$ within a certain range should be fulfilled to achieve an efficient conversion of the optical excitation energy of the donor to the acceptor. The strong 6th power dependence of FRET efficiency on $r$ makes this process ideally suited for probing separation distances in the range of $1 \div 10 \mathrm{~nm}$. Thus, FRET uses for development of sensitive tools 
for the investigation of conformational changes in proteins [2, 3], on-off switched optical sensors [4-7], and molecular bioimaging including single molecule fluorescence microscopy [8].

Numerous reports published in the past few years were focused on the effective use of colloidal quantum dots (QDs) as donors in various FRET-based studies and sensing demonstrations [9]. QDs demonstrate broad excitation and size-tunable photoluminescence spectra with narrow emission band allowing simultaneous excitation of particles within a certain size range at a single wavelength. QDs also reveal exceptional photochemical stability and high quantum yield. Additionally, QD surface can be modified by various ligands for an efficient binding of biological molecules including biopolymers (like DNA and peptides) [10].

At the same time, the classical Förster theory [1] treats donors and acceptors as points considering energy transfer within a single donor-acceptor pair. The application of this approach to the systems involving QDs is limited due to a relatively large size of QDs (one order of magnitude larger than the typical size of organic fluorophores). This results in a multiple energy transfer configurations with one excitation source in the QD-dye systems. The quantitative description becomes even more challenging if the system consists of several randomly distributed donors and acceptors.

In the present paper we contribute to the consideration of multiple energy transfer configurations for the case of randomly distributed QDs and dye. For this purposes a model system consisting of CdTe quantum dots randomly distributed within the polymer particles bound to spectrally suitable dye molecules (erythrosin B) was developed. Details of the synthesis of colloidal particles based on chitosan and chondroitin sulfate, doped with dye [11] and quantum dots [12] were published previously. FRET efficiency was investigated by means of fluorescence spectroscopy, including time-resolved technique. The obtained data were evaluated by the advanced Förster theory for several randomly distributed donors and acceptors [1]; the average distances between donor and acceptor as well as local concentration of fluorophores immobilized in particles were determined.

\section{EXPERIMENTAL}

\subsection{Materials}

Water-soluble quantum dots CdTe stabilized by a carboxyl groups with emission peak at $527 \mathrm{~nm}$ in water were supplied by PlasmaChem, Germany. The core size of QD was determined as $2.2 \mathrm{~nm}$ based on data [13] and then the molar extinction coefficient $\varepsilon_{481}$ was estimated as $5.5 \times 10^{4} \mathrm{M}^{-1} \mathrm{~cm}^{-1}$ [14].

Low-viscosity chitosan (aminopolysaccharide, 2-amino-2-deoxy- $\beta$-D-glucan) from shrimp shells, sodium salts of chondroitin sulfate A ( $\beta$-glucuronic acid- $(1 \rightarrow 3)-\mathrm{N}$-acetyl- $\beta$-galactosamine-4sulfate-(1 $\rightarrow 4)$ ) from bovine trachea, organic dye erythrosin B (Figure 1) and flavin 
mononucleotide (FMN) were supplied by Sigma-Aldrich. All chemicals were used without further purification. Stock solution of $0.1 \% \mathrm{w} / \mathrm{v}$ chitosan was prepared in $0.15 \mathrm{M}$ acetate buffer, $\mathrm{pH}$ 5.5; double-distillated was used to prepare stock solutions of $0.1 \% \mathrm{w} / \mathrm{v}$ chondroitin sulfate, $10^{-4} \mathrm{M}$ QD and $10^{-3} \mathrm{M}$ dye.

\subsection{Synthesis of biopolymer particles with CdTe QDs}

The method used for the synthesis of biopolymer particles doped with CdTe QDs from chitosan and chondroitin sulfate was similar to the described previously [15]. Chitosan solution of $0.1 \% \mathrm{w} / \mathrm{v}$ concentration was pre-cleaned from insoluble impurities by filtration through a paper filter prior to the adding of the QDs stock solution. The mixture was stirred for at least two hours to provide binding of carboxyl groups of quantum dots stabilizer with amino groups of chitosan. Afterwards the chondroitin sulfate solution of $0.1 \% \mathrm{w} / \mathrm{v}$ concentration was added dropwise to the chitosan-QDs solution to obtain polymer particles. The mixture was stirred for two hours. Volume ratio for polycation (chitosan) and polyanion (chondroitin sulfate) solutions was 2:1. The molecular polymer fraction was separated from the colloid solution by 5 min centrifugation at $14,500 \mathrm{rpm}$ and $25^{\circ} \mathrm{C}$. After the elimination of the molecular fraction the precipitate was ultrasonically resuspended for $30 \mathrm{~min}$ in an unbuffered solvent with $\mathrm{pH}$ 5.2. After the latter procedure the $\mathrm{pH}$-value of the solution containing biopolymer particles with immobilized CdTe QDs grew up to 5.6. Finally, the dye stock solution was added to the colloidal solution of QD-doped biopolymer particles. The mixture was maintained for $15 \mathrm{~min}$ to achieve the dye-biopolymer binding upon sorption-desorption equilibrium. The apparent dye concentrations in solution was varied within the range of $(0.3-7.3) \times 10^{-6} \mathrm{M}$.

\subsection{Dynamic light scattering and $\zeta$-potential}

The particle size distribution in the investigated biopolymer particles solutions was derived from the dynamic light scattering (DLS) data obtained by three repetitive measurements for each sample using a Zetasizer Nano ZS instrument (Malvern Instruments Ltd.). The analysis of the autocorrelation function was carried out in approximation of solid spherical particles. $\zeta$-potential measurements were performed on the Delsa Nano instrument (Beckman Coulter); each value represents an average of three repetitive runs.

\subsection{Transmission electron and confocal laser scanning microscopy}

Transmission electron microscope (TEM) HT7700 (Hitachi, Japan) and confocal laser scanning microscope (CLSM) LSM 780 NLO (Zeiss, Germany) prepared the images of biopolymer particles. The samples were prepared by dropping of diluted solutions of biopolymer particles with immobilized CdTe QDs onto 300-mesh carbon coated copper grids with a thin carbon film and 
cover slips, for TEM and CLSM studies, respectively; and then samples were dried. The samples were observed by CLSM with excitation wavelengths of $405 \mathrm{~nm}$. CLSM images were processed using the software ImageJ (National Institutes of Health, USA).

\subsection{Steady-state and time-resolved measurements}

The absorption spectra of CdTe immobilized in biopolymer particles with increasing erythrosin B in were detected using Lambda 35 spectrophotometer (Perkin Elmer). The fluorescence spectra were measured on a Fluorolog 3-22 spectrofluorometer (Horiba Jobin Yvon). Examined solutions were excited at the wavelength of $373 \mathrm{~nm}$. The obtained fluorescence spectra were corrected for the reabsorption, if necessary, and for sensitivity of the recording system.

The time-resolved measurements were performed using the same spectrofluorometer equipped with the FluoroHub B timing module (Horiba Jobin Yvon) enabling time-correlated single-photon counting. The excitation source was a pulsed laser diode NanoLED N-370 with the peak wavelength at $373 \mathrm{~nm}$ operating at $100 \mathrm{MHz}$ with an optical pulse duration <1.2 ns. The deconvolution procedure and the analysis of the fluorescence decays were performed by means of DAS6 software using $\chi^{2}$ statistical criteria. The decay of biopolymer-immobilized QDs both in the absence and in the presence of the dye was collected at $536 \mathrm{~nm}$ and fitted by the sum of three exponentials: $I(t)=\sum A_{i} \exp \left(-t / \tau_{i}\right)$. The average lifetime of QDs was calculated as $\langle\tau\rangle=\sum f_{i} \tau_{i}$, where $f_{i}$ and $\tau_{i}$ are the relative amplitudes and lifetime of the $i$ th decay component, respectively.

The fluorescence quantum yield of the QDs immobilized in particles was obtained by calibration with FMN in water (0.26) as a reference. Standard quartz cells with cross sections of $10 \times 10 \mathrm{~mm}$ were used to investigate solutions for L-geometry of excitation. Measurements were performed at room temperature.

\section{THEORY OF FÖRSTER RESONANCE ENERGY TRANSFER}

According to the theory of Förster [1] for single donor-acceptor transfer, the rate constant $\left(k_{\mathrm{ET}}\right)$ is determined as follows:

$$
k_{\mathrm{ET}}(r)=\frac{1}{\tau_{\mathrm{D}}}\left(\frac{R_{0}}{r}\right)^{6},
$$

where $\tau_{\mathrm{D}}$ is the intrinsic lifetime of the donor, $r$ is the distance between donor and acceptor. $R_{0}$ is the critical distance for which excitation transfer and spontaneous deactivation of the donor are of equal probability:

$$
R_{0}=\left(\frac{9000 \ln 10}{128 \pi^{5} n^{4} N} \kappa^{2} \Phi_{\mathrm{D}} J_{\mathrm{DA}}\right)^{1 / 6}
$$


where $\kappa^{2}$ is the dipole orientation factor, $\Phi_{\mathrm{D}}$ is the fluorescence quantum yield of donor, $n$ is solvent refractive index, $N$ is Avogadro's number. The value of $J_{\mathrm{DA}}$ provides the degree of the spectral overlap between donor fluorescence and acceptor absorption:

$$
J_{\mathrm{DA}}=\int I_{\mathrm{D}}(\tilde{v}) \varepsilon_{\mathrm{A}}(\tilde{v}) \tilde{v}^{-4} d \tilde{v} / \int I_{\mathrm{D}}(\tilde{v}) d \tilde{v},
$$

where $I_{\mathrm{D}}(\tilde{v})$ is the fluorescence intensity of the donor, $\varepsilon_{\mathrm{A}}(\tilde{v})$ is the molar extinction coefficient of the acceptor. From steady-state and time-resolved experiments, one can obtain the FRET efficiency as

$$
E=1-\frac{I_{\mathrm{DA}}}{I_{\mathrm{D}}}=1-\frac{\left\langle\tau_{\mathrm{DA}}\right\rangle}{\left\langle\tau_{\mathrm{D}}\right\rangle},
$$

where $I_{\mathrm{D}},\left\langle\tau_{\mathrm{D}}\right\rangle$ and $I_{\mathrm{DA}},\left\langle\tau_{\mathrm{DA}}\right\rangle$ is fluorescence intensity and actual average lifetime of donor in absence (subscript D) and presence (subscript DA) of acceptor, respectively. FRET efficiency relates with D-A distance through the simple equation:

$$
E=\frac{1}{1-\left(\frac{r}{R_{0}}\right)^{6}} .
$$

The most common case of the energy transfer can be represented by randomly distributed multiple donors and acceptors. Upon the assumptions of identical properties of all donors and all acceptors (single donor and single acceptor types are present in the system), FRET efficiency can be estimated by the following relation [1]:

$$
E=\sqrt{\pi} \frac{C_{\mathrm{A}}}{C_{0}} \exp \left(\frac{C_{\mathrm{A}}}{C_{0}}\right)^{2}\left[1-\operatorname{erf}\left(\frac{C_{\mathrm{A}}}{C_{0}}\right)\right],
$$

where $C_{\mathrm{A}}$ is the acceptor concentration, $C_{0}$ is the critical concentration which is related to the critical transfer distance $R_{0}: C_{0}=3000 / 4 \pi N R_{0}^{3}$. This corresponds to an average of one acceptor molecule in a sphere with the radius $R_{0}$. Taking $\left(C_{\mathrm{A}} / C_{0}\right)=\left(R_{0} / r\right)^{3}$ into account, one can arrange Eq. (6) in terms of distances:

$$
E=\sqrt{\pi}\left(\frac{R_{0}}{r}\right)^{3} \exp \left(\frac{R_{0}}{r}\right)^{6}\left[1-\operatorname{erf}\left(\frac{R_{0}}{r}\right)^{3}\right]
$$

Taking into consideration the fact, that the concentration of acceptor significantly exceeds the concentration of donors, the contribution of the donor-donor energy transfer can be assumed as negligible. Here the approximation $C_{\mathrm{A}}>C_{\mathrm{D}}\left(C_{\mathrm{D}}\right.$ is the donor concentration) is taken into account to eliminate a donor-donor energy transfer. In both models, Eqs. (5) and (7), the theory 
relates FRET efficiency with the inter-fluorophore distance $r$, however the functional relationships are significantly different, especially for $r>R_{0}$.

\section{RESULTS}

\subsection{Morphology and $\zeta$-potential of QD-doped particles}

The CLSM study confirmed the presence of fluorescent polymer particles (Figure 2a). The lower limit of the concentration of the polymer particles estimated by processing of a CLSM imaging of a single layer was $2 \times 10^{-9} \mathrm{M}$. The dried droplet may consist of multiple (several) layers, therefore the particle concentration of the order of $10^{-8} \mathrm{M}$ can be accepted for further rough estimates. TEM images (Figure 2b) reveal the localization of QDs in polymer particles. A single polymer particle can contain about 200 quantum dots. The particle size after drying was reduced upto $80 \mathrm{~nm}$, while the average size of the polymer particles in the colloidal solution was $330 \pm 23 \mathrm{~nm}$. The $\zeta$-potential was $+42 \mathrm{mV}$, that corresponds to the requirement of colloidal stability of particles.

\subsection{Steady-state and time-resolved measurements}

To meet one of the most principal requirements for FRET observation an acceptable overlap of the donor fluorescence and the acceptor absorption bands should be provided (Figure 3). The values of the overlap integral $J_{\mathrm{DA}}$ obtained by Eq. (3) and the critical transfer distance $R_{0}$ obtained by Eq. (2) amounted 3.3 $\times 10^{-13} \mathrm{M}^{-1} \mathrm{~cm}^{-3}$ and $4.75 \mathrm{~nm}$, respectively. Dipole orientation factor was supposed to be $\kappa^{2}=2$ / 3 in the fast rotating dipole approximation [1]. In the case of D-A binding through a biopolymer particle the model of statistically distributed immobile dipoles is reasonable giving $\kappa^{2}=0.476$ [1]. The value of $R_{0}$ derived within this approximation was $4.50 \mathrm{~nm}$ that is quite similar to the result obtained in the fast rotating dipole approximation. The value of 1.337 was taken the for the refractive index $n$ of the chitosan solution [16]. The determined fluorescence quantum yield $\Phi_{\mathrm{D}}$ of CdTe QDs immobilized in biopolymer particles was 18.9\%.

Another important condition of FRET consists in the proximity of donors and acceptors - the interfluorophore distance $r$ should not be much more than critical transfer distance. The apparent QDs concentration obtained by spectral measurements (Figure 4) was equal to $2.2 \times 10^{-6} \mathrm{M}$. The apparent dye concentration at peak addition was $7.3 \times 10^{-6} \mathrm{M}$, that approximately three orders of magnitude less than the critical concentration $\left(3.7 \times 10^{-3} \mathrm{M}\right)$. However, the proximity to critical concentration is very essential for the FRET observation. The compliance of this condition was provided by the immobilization of QDs (as donors) within the polymer particles and binding of dye molecules (as acceptors) to polymer. Due to the fact that the local concentration of fluorophores within the 
particles is much greater than the apparent concentration in solution depictured in Figure 4, concentration becomes close to the critical one.

Despite the fact that the ratio $C_{\mathrm{A}}>C_{\mathrm{D}}$ was not always fulfilled within the framework of our experiment, the Förster distance estimation for the donor-donor transfer (3.72 nm) turned out to be less than that for the donor-acceptor transfer $(4.75 \mathrm{~nm})$. That suggests the possibility of the Förster theory application in our case.

The results of steady-state (quenching of donor fluorescence and enhancement of acceptor fluorescence) and time-resolved (decrease of donor lifetime) measurements for the system including QDs ensemble and dye molecules represented in Figure 5a,b evident the occurrence of FRET. The quantitative characteristics of these dependences were determined in terms of Stern-Volmer plots (Figure 6) revealing a linear relationship throughout the concentration range of the acceptor studied in the case of time-resolved measurements. It is commonly recognized that the time-resolved spectroscopy technique is more appropriate for FRET studies, since the data obtained by steadystate spectroscopy are overestimated due to the presence of additional static quenching through the formation of non-fluorescent complexes [17]. The efficiencies of the QD-dye energy transfer calculated according to Eq. (4) turned out to be quite high reaching the value of $80 \%$.

Figure 7 shows the calculated transfer efficiency as a function of D-A distance for two FRET models corresponding to a single donor-acceptor transfer from Eq. (5) and randomly distributed multiple donors and acceptors from Eq. (7). As predicted by the Förster theory, a 50\% probability of energy transfer at the distance $r=R_{0}$ is realized for the first model. For the case of multiply dipoles, the same efficiency is achieved at $r \approx 1.3 R_{0}$. The best agreement between experimental data and theoretical calculations was demonstrated for the model of randomly distributed dipoles by varying the acceptor local concentration in a polymer particle. The local concentration of dye covering the range $(0.3-6.6) \times 10^{-3} \mathrm{M}$ found to be 900 times greater than the apparent concentration in solution.

Curves 1, 2 in Figure 7 reveal the region with maximum sensitivity of the D-A distance measurement by FRET technique. The linear part of these curves corresponds the transfer efficiency range of about 20-80\%. This interval can be transformed into the distance variation range: $r=(0.79 \div 1.26) R_{0}$ for the case of single donor-acceptor transfer and $r=(0.91 \div 1.90) R_{0}$ for randomly distributed multiple donors and acceptors. The latter have 2-fold range that corresponds to the interval from 4 to $9 \mathrm{~nm}$ for our system. 


\section{CONCLUSIONS}

Spectrally appropriate QDs (CdTe, emission peak $536 \mathrm{~nm}$ ) and dye (erythrosin B, absorption peak $536 \mathrm{~nm}$ ) were immobilized in chitosan-based biopolymer particles with average sizes of about $0.3 \mu \mathrm{m}$. A high local concentration of fluorophores within the particles provides inter-fluorophore distances within an appropriate range favoring an effective energy transfer between QD ensembles and dye molecules. The efficiency of the energy transfer reaching the value of $80 \%$ was measured by both steady-state and time-resolved spectroscopic techniques. The dependence of the FRET efficiency on acceptor concentration was successfully described within the framework of the Förster model extended for the case of multiple energy transfer configurations. The local concentration of dye within the particles covering the range $(0.3-6.6) \times 10^{-3} \mathrm{M}$ found to be 900 times greater than the apparent concentration in solution. The developed multiple donor-acceptor system can be used as FRET-sensing element, working in the D-A distance of $4 \div 9 \mathrm{~nm}$.

\section{ACKNOWLEDGMENTS}

The authors are grateful to the Tomsk Regional Center for Collective Use of the Tomsk State University, to I. Lapin for help in CLSM studies, to the Center for Collective Use of the Krasnoyarsk Scientific Center of the Siberian Branch of the Russian Academy of Sciences, to M. Volochaev for her help in TEM studies, to the Laboratory of Biotechnology of New Biomaterials of Siberian Federal University (Prof. T. G. Volova) for kindly providing the analyzer Zetasizer Nano ZS.

\section{REFERENCES}

[1] Förster, T. "Transfer mechanisms of electronic excitation energy," Radiat. Res. Suppl. 2, 326-339 (1960).

[2] Heyduk, T. "Measuring protein conformational changes by FRET/LRET," Curr. Opin. Biotechnol. 13, 292-296 (2002).

[3] Medintz, I. L., Pons, T., Susumu, K., Boeneman, K., Dennis, A. M., Farrell, D., Deschamps, J. R., Melinger, J. S., Bao, G., Mattoussi, H. "Resonance energy transfer between luminescent quantum dots and diverse fluorescent protein acceptors," J. Phys. Chem. C. 113, 18552-18561 (2009).

[4] Clapp, A. R., Medintz, I. L. and Mattoussi, H. "Förster resonance energy transfer investigations using quantum-dot fluorophores," ChemPhysChem 7, 47-57 (2006). 
[5] Liu, C.-W. and Chang, H.-T. "Protein-conjugated quantum dots for detecting trypsin and trypsin inhibitor through fluorescence resonance energy transfer," Open Anal. Chem. J. 1, 1-6 (2007).

[6] Clapp, A. R., Medintz, I. L., Mauro, J. M., Fisher, B. R., Bawendi, M. G. and Mattoussi, H. "Fluorescence resonance energy transfer between quantum dot donors and dye-labeled protein acceptors," J. Am. Chem. Soc. 126, 301-310 (2004).

[7] Dennis, M., Rhee, W. J., Sotto, D., Dublin, S. N. and Bao, G. "Quantum dot-fluorescent protein fret probes for sensing intracellular pH," ACS Nano 6(4), 2917-2924 (2012).

[8] Roy, R., Hohng, S. and Ha, T. "A practical guide to single-molecule FRET," Nat. Methods 5(6), 507-516 (2008).

[9] Medintz, I. L. and Mattoussi, H. "Quantum dot-based resonance energy transfer and its growing application in biology," Phys. Chem. Chem. Phys. 11, 17-45 (2009).

[10] Rosenthal, S. J., Chang, J. C., Kovtun, O. McBride, J. R. and Tomlinson, I. D. "Biocompatible quantum dots for biological applications," Chem. Biol. 18(1), 10-24 (2011).

[11] Slyusareva, E., Gerasimova, M., Slabko, V., Abuzova, N., Plotnikov, A. and Eychmüller, A. "Synthesis and characterization of chitosan-based polyelectrolyte complexes doped with xanthene dyes," ChemPhysChem 16(18), 3997-4003 (2015).

[12] Abuzova, N. V., Gerasimova, M. A., Slabko, V. V. and Slyusareva, E. A. "Synthesis and characterization of chitosan-based polyelectrolyte complexes, doped by quantum dots," Proc. SPIE 9810, 1-6 (2015).

[13] Rogach, A. L., Franzl, T., Klar, T. A., Feldmann, J., Gaponik, N., Lesnyak, V., Shavel, A., Eychmüller, A., Rakovich, Y.P. and Donegan, J. F. "Aqueous synthesis of thiol-capped CdTe nanocrystals: State-of-the-art," J. Phys. Chem. C. 111, 14628-14637 (2007).

[14] Yu, W., Qu, L., Guo, W. and Peng, X. "Experimental determination of the extinction coefficient of CdTe, CdSe, and CdS nanocrystals," Chem. Mater. 15, 2854-2860 (2003).

[15] Slyusarenko, N. V., Gerasimova, M. A., Slabko, V. V. and Slyusareva, E. A. "Thermal sensitivity of water-soluble CdTe and CdSe/ZnS quantum dots embedded in biopolymer submicron particles," Russ. Phys. J. 60(3), 477-484 (2017).

[16] Koralewski, M., Bodek, K. H. and Marczewska K., "Optical properties of chitosan in aqueous solution," Polish Chitin Society, Monograph 11, 29-39 (2006).

[17] Lakowicz, J. R., [Principles of fluorescence spectroscopy], Springer Science \& Business Media, New York (2013). 


\section{FIGURE CAPTURES}

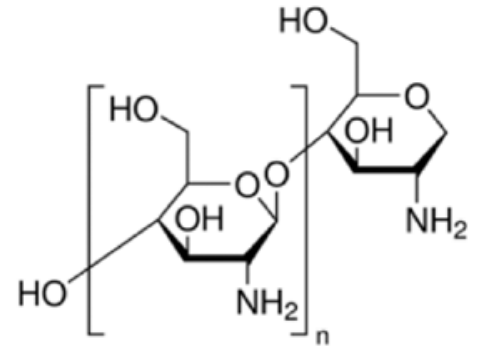

Chitosan

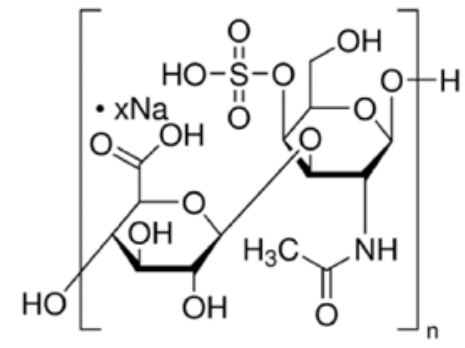

Chondroitin sulfate<smiles>O=c1c(I)cc2c(-c3ccccc3O[O-])c3cc(I)c(O[Na])c(I)c3oc-2c1I</smiles>

Erythrosin B

Figure 1. Molecular structures of biopolymers and dye.

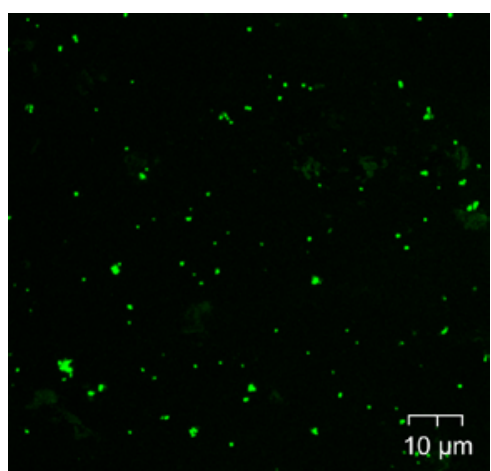

(a)

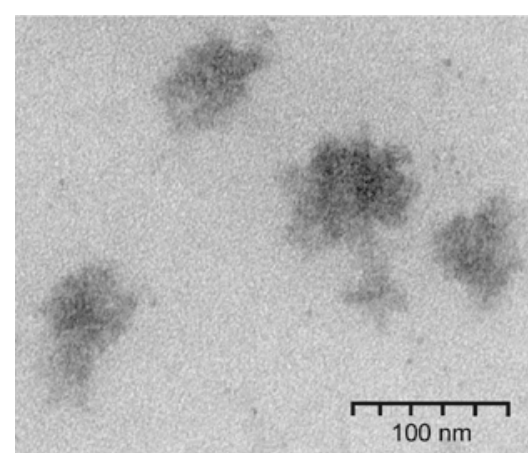

(b)

Figure 2. CLSM (a) and TEM (b) images of QDs-doped biopolymer particles.

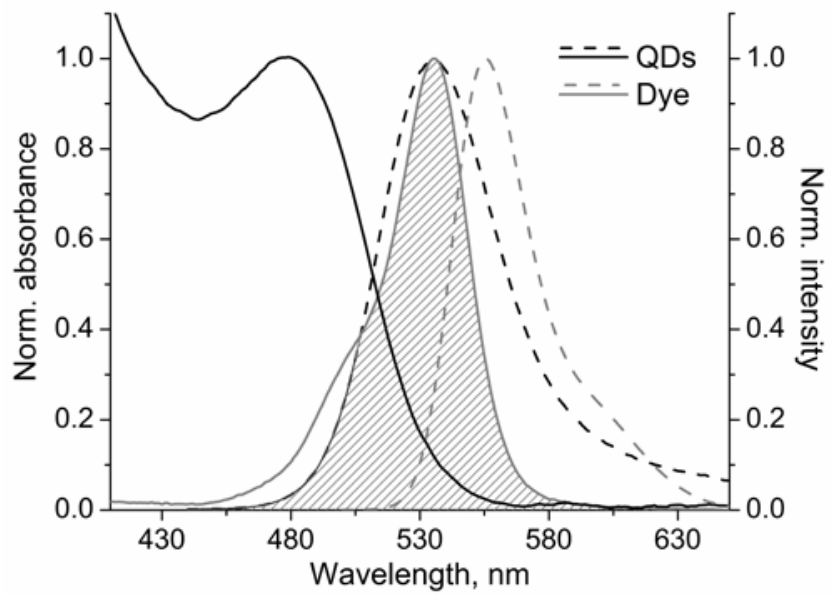

Figure 3. Absorption and fluorescence spectra of biopolymer-immobilized CdTe QD and erythrosin B. 


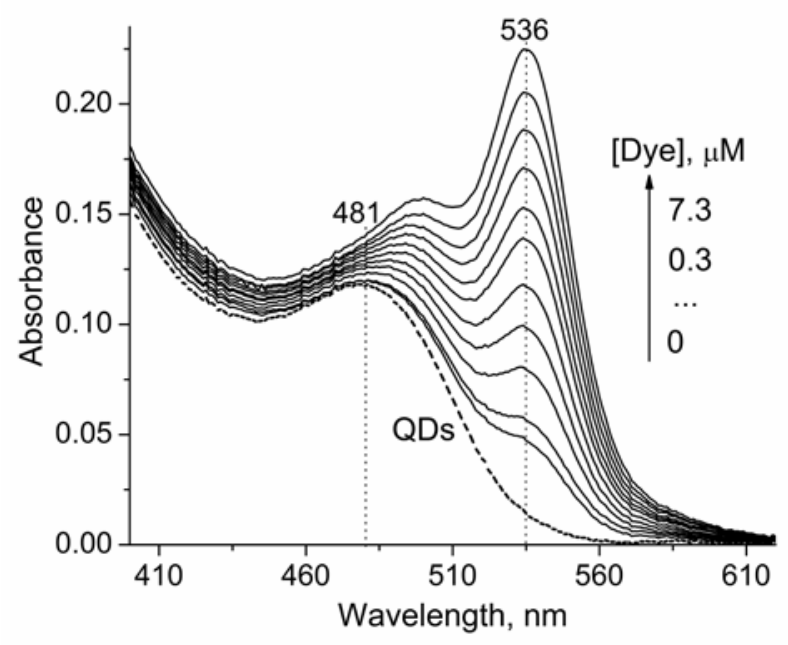

Figure 4. Absorption spectra of QDs (dotted line) immobilized in biopolymer particles and bound dye (solid lines) of different concentration.

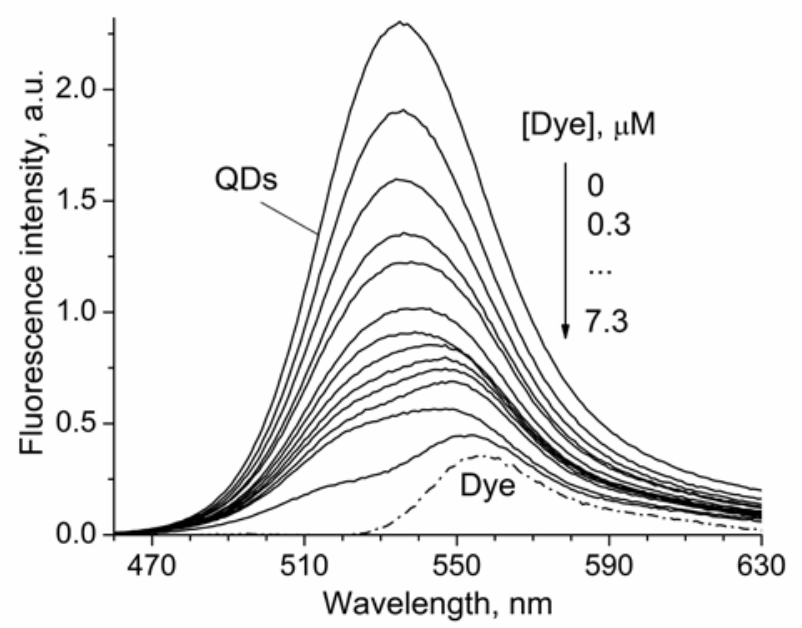

(a)

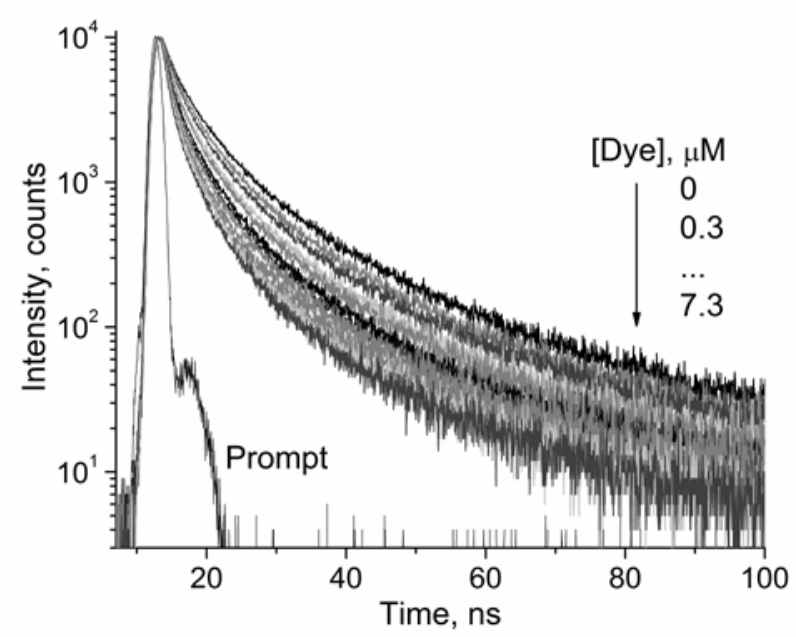

(b)

Figure 5. The fluorescence steady-state spectra (a) and time-resolved decays (b) of biopolymer-immobilized QDs with increasing dye concentration. 


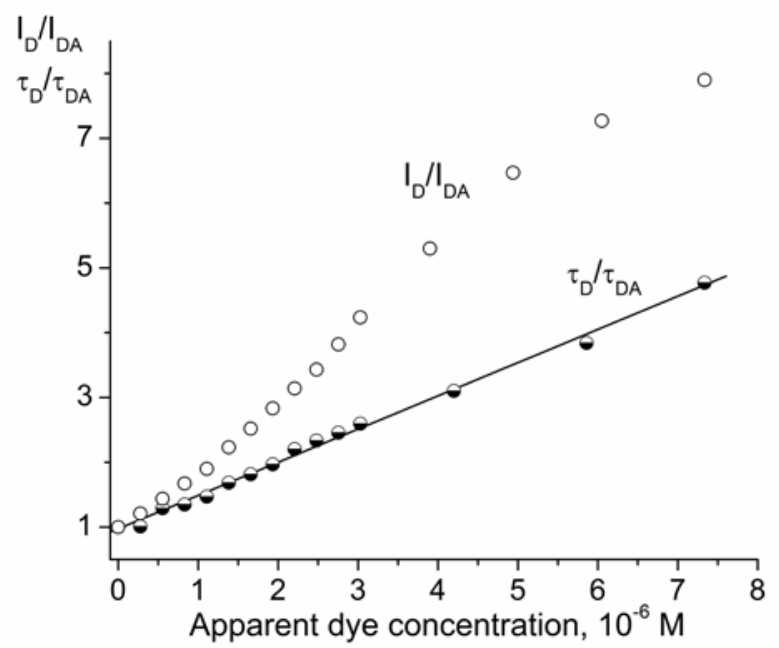

Figure 6. Stern-Volmer plots of $I_{\mathrm{DA}} / I_{\mathrm{D}}$ and $\tau_{\mathrm{DA}} / \tau_{\mathrm{D}}$ vs. apparent concentration of dye.

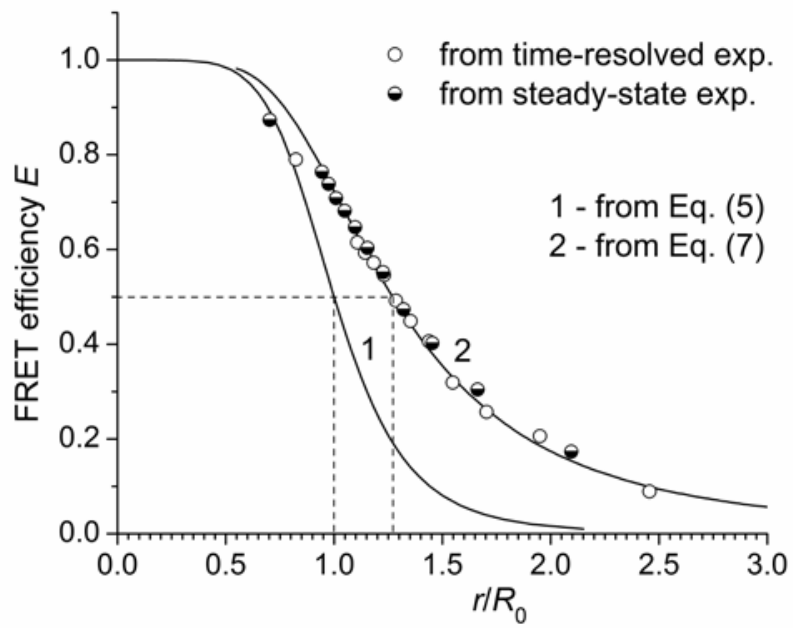

Figure 7. FRET efficiency vs. the distance between the donors (QDs) and acceptors (dye). 